\title{
REMARKS ON THE GAUSS-LUCAS THEOREM IN HIGHER DIMENSIONAL SPACE
}

\author{
A. W. GOODMAN
}

\begin{abstract}
A recent paper by J. B. Diaz and Dorothy Browne Shaffer extends the Gauss-Lucas Theorem to $n$-dimensional Euclidean space. The authors leave open certain natural questions concerning the existence of "zeros of the derivative". This paper answers three such questions, and suggests several other questions for further investigation.
\end{abstract}

1. Introduction. The classical Gauss-Lucas Theorem states that if $H$ is the closed convex hull of the zeros of $P(z)$, a polynomial in one complex variable, then all the zeros of $P^{\prime}(z)$ must also lie in $H$. A proof of this beautiful theorem may be found in many places (see, for example, the book by Morris Marden [3]).

It is natural to look for extensions of this theorem to $E_{n}$, the $n$-dimensional Euclidean space consisting of all points $P:\left(x_{1}, x_{2}, \ldots, x_{n}\right)$ where the coordinates are real numbers. Recently, Diaz and Shaffer [1] found an interesting extension which we now describe. In their work they use the symbol $w=\left(w_{1}, w_{2}, \ldots, w_{n}\right)$ for a point in $E_{n}$, but, since this notation suggests that $w_{k}$ may be a complex number, we take the liberty of altering their notation. Let

Let $P_{j}:\left(x_{1}^{(j)}, x_{2}^{(j)}, \ldots, x_{n}^{(j)}\right), j=1,2, \ldots, m$, be $m$ distinct points in $E_{n}$.

$$
r_{j}=\left|P P_{j}\right|=\left[\sum_{k=1}^{n}\left(x_{k}-x_{k}^{(j)}\right)^{2}\right]^{1 / 2}
$$

be the usual Euclidean distance from $P$ to $P_{j}$. With each fixed point $P_{j}$, we associate some particular function $f_{j}\left(r_{j}\right)$, which may be regarded as a generalized distance. Thus, we require that if $r \geqslant 0$, then $f_{j}^{\prime}(r)>0$ for $j=1$, $2, \ldots, m$. We could also demand that $f_{j}(r)>0$, but it is conveneint to avoid this additional restriction. In most cases this can be achieved by adding to $f_{j}(r)$ a suitable constant. We consider the function

$$
F(\mathbf{x})=\sum_{j=1}^{m} f_{j}\left(r_{j}\right)
$$

where (throughout this paper) $\mathbf{x}$ is a convenient shorthand for the variable point $P:\left(x_{1}, x_{2}, \ldots, x_{n}\right)$. The function $F(\mathbf{x})$ defined by equation (2) may be

Presented to the Society, January 23, 1975; received by the editors October 10, 1974.

AMS (MOS) subject classifications (1970). Primary 26-00; Secondary 26A78, 26A90, 30A06, 30A92.

Key words and phrases. Polynomials, zeros of the derivative, Euclidean $n$-space, gradient, Gauss-Lucas Theorem. 
regarded as a generalized polynomial because, if each $f_{j}(r)$ is the function $\log r$, and $P(z)=\left(z-z_{1}\right) \cdots\left(z-z_{m}\right)$, then $F(\mathbf{x})=\log |P(z)|$ in $E_{2}: z$ $=x_{1}+i x_{2}$. Further, if $P^{\prime}(z)=0$, and $z$ is not a zero of $P(z)$, then

$$
\begin{aligned}
\nabla F(\mathbf{x}) & \equiv \sum_{j=1}^{m} \frac{\partial}{\partial x_{1}} \log \left|z-z_{j}\right| \mathbf{e}_{1}+\sum_{j=1}^{m} \frac{\partial}{\partial x_{2}} \log \left|z-z_{j}\right| \mathbf{e}_{2} \\
& \left.=\sum_{j=1}^{m} \frac{z-z_{j}}{\left|z-z_{j}\right|^{2}}=\sum_{j=1}^{m} \frac{1}{\frac{P^{\prime}(z)}{P(z)}}\right)=0 .
\end{aligned}
$$

Here, of course, $\mathbf{e}_{k}$ denotes a unit vector in the direction of the $x_{k}$ axis. With these notations and motivations, we can state

Theorem A (Diaz-Shaffer [1]). Let $P_{1}, \ldots, P_{m}$ be $m$ distinct points in $E_{n}$, and with each point $P_{j}$ associate a function $f_{j}(r)$ for which $f^{\prime}(r)$ is positive and continuous for $r>0$. Set $r_{j}=\left|P P_{j}\right|$ and define $F(\mathbf{x})$ by equation (2). If $\mathbf{x}$ is a zero of the vector function

$$
\nabla F(\mathbf{x}) \equiv \sum_{k=1}^{n} \frac{\partial F}{\partial x_{k}} \mathbf{e}_{k}
$$

then $\mathbf{x}$ lies in $H$, the closed convex hull of the set of points $P_{1}, P_{2}, \ldots, P_{m}$.

This theorem tells us where a zero of "the derivative" $\nabla F(\mathbf{x})$ must lie, if there is one. But it leaves open the question of whether $\nabla F(\mathbf{x})$ has any zeros, and, if so, how many. We will prove that, under suitable conditions, there is always at least one zero, and we will give a partial answer to the question "How many?".

2. Answers and proofs. We can avoid unnecessary radicals by introducing new functions $g_{j}(r)$. Indeed, for $r>0$ we set $g_{j}(r)=f_{j}\left(r^{1 / 2}\right), j=1,2, \ldots, m$. It is easy to see that if $g_{j}^{\prime}(r)$ is positive and continuous for $r>0$, then so is $f_{j}^{\prime}(r)$, and conversely. However, it should be noted that this assertion cannot be extended to include $r=0$. Using these new functions, we have

$$
F(\mathbf{x})=\sum_{j=1}^{m} f_{j}\left(r_{j}\right)=\sum_{j=1}^{m} g_{j}\left(r_{j}^{2}\right)=\sum_{j=1}^{m} g_{j}\left(\sum_{k=1}^{n}\left(x_{k}-x_{k}^{(j)}\right)^{2}\right) .
$$

THEOREM 1. Let $g_{j}^{\prime}(r)$ be continuous and positive for $r \geqslant 0(j=1,2, \ldots, m)$, and let $P_{1}, P_{2}, \ldots, P_{m}$ be $m(\geqslant 2)$ distinct points in $E_{n}$. If $F(\mathbf{x})$ is defined by equation (5), then $\nabla F(\mathbf{x})$ always has at least one zero in $H$.

Proof. Our proof uses the Brouwer fixed point theorem [2, p. 453] and, consequently, we must assume that $g_{j}^{\prime}(r)$ is continuous on the close ray $r \geqslant 0$. We will return to this additional hypothesis in $\$ 3$.

For each term in equation (5), we have

$$
\nabla f_{j}\left(r_{j}\right)=\nabla g_{j}\left(r_{j}^{2}\right)=2 g_{j}^{\prime}\left(r_{j}^{2}\right) \sum_{k=1}^{n}\left(x_{k}-x_{k}^{(j)}\right) \mathbf{e}_{k} .
$$

Consequently, 


$$
\begin{aligned}
\nabla F(\mathbf{x}) \sum_{j=1}^{m} \nabla g_{j}\left(r_{j}^{2}\right) & =2 \sum_{j=1}^{m}\left(\sum_{k=1}^{n} g_{j}^{\prime}\left(r_{j}^{2}\right)\left(x_{k}-x_{k}^{(j)}\right) \mathbf{e}_{k}\right) \\
& =2 \sum_{k=1}^{n}\left(\sum_{j=1}^{m} g_{j}^{\prime}\left(r_{j}^{2}\right)\left(x_{k}-x_{k}^{(j)}\right)\right) \mathbf{e}_{k} .
\end{aligned}
$$

For brevity, set $A_{j}=g_{j}^{\prime}\left(r_{j}^{2}\right)$, and $B_{j}=A_{j} / \sum_{\alpha=1}^{m} A_{\alpha}$. Assume now that $\nabla F(\mathbf{x})=\mathbf{0}$. Then each component must be zero, and (7) leads to the system of equations

$$
x_{k}=\sum_{j=1}^{m} B_{j} x_{k}^{(j)}, \quad k=1,2, \ldots, n,
$$

where $B_{j}>0$ for $r \geqslant 0$, and $\sum_{j=1}^{m} B_{j}=1$.

We note, in passing, that equation set (8) already contains the proof of the Diaz-Shaffer Theorem since the conditions on $B_{j}$ imply that $\left(x_{1}, x_{2}, \ldots, x_{n}\right)$ is in the closed convex hull of $P_{1}, P_{2}, \ldots, P_{m}$.

To see that the system (8) always has a solution, we replace the left side by the coordinates $x_{k}^{*}$ of a point $P_{k}^{*}\left(x_{1}^{*}, x_{2}^{*}, \ldots, x_{n}^{*}\right)$, and obtain

$$
x_{k}^{*}=\sum_{j=1}^{m} B_{j} x_{k}^{(j)}, \quad k=1, \ldots, n,
$$

where the $B_{j}$ are functions of $P\left(x_{1}, x_{2}, \ldots, x_{n}\right)$. Then the equation set (9) can be regarded as a mapping that carries $P$ into $P^{*}$. Since this transformation is continuous on $H$ and carries each point of $H$ into a point of $H$, then by the Brouwer fixed point theorem, there is at least one fixed point of the transformation. Since this fixed point is a solution of the system (8), it follows that $\nabla F(\mathbf{x})$ always has at least one zero.

Returning to the original Gauss-Lucas Theorem, we note that if $P(z)$ is a polynomial of degree $m$, then $P^{\prime}(\mathrm{z})$ will always have $m-1$ zeros when counted in accordance with their multiplicities. Is there an analogue for $\nabla F(\mathbf{x}) \equiv G(\mathbf{x}) ?$

We first need a

Definition. The point $P$ is a zero of $G(\mathbf{x}) \equiv G\left(x_{1}, x_{2}, \ldots, x_{n}\right)$ of order $k$ if, at $P$, the function and all its partial derivatives of order less than $k$ vanish, while at least one partial derivative of order $k$ does not vanish at $P$.

For example, $G(\mathbf{x})=x_{1}^{k}+x_{2}^{k}+x_{3}^{k}$ has a $k$ th order zero at $(0,0,0)$ in $E_{3}$. With this agreement we have

THEOREM 2. For arbitrary natural numbers $n$ and $m$, it is possible to select the "distance" functions $g_{j}(r)$ so that $\nabla F(\mathbf{x})$ has only one zero, and this zero is a simple zero.

Proof. For $j=1,2, \ldots, m$ set $g_{j}(r)=r$. Then $A_{j} \equiv g_{j}^{\prime}\left(r_{j}^{2}\right)=1$, and, hence, the coefficients in the system (8) are all $1 / \mathrm{m}$. Consequently, the point $P\left(x_{1}, x_{2}, \ldots, x_{n}\right)$ determined by this system is unique. To see that $P$ is a simple zero, observe that, in this case,

$$
\nabla F(\mathbf{x})=2 \sum_{k=1}^{n}\left(\sum_{j=1}^{m}\left(x_{k}-x_{k}^{(j)}\right)\right) \mathbf{e}_{k} \equiv \sum_{k=1}^{n} G_{k}(\mathbf{x}) \mathbf{e}_{k} .
$$


But for the first component $G_{1}$ we have

$$
\frac{\partial G_{1}(\mathbf{x})}{\partial x_{1}}=2 \frac{\partial}{\partial x_{1}} \sum_{j=1}^{m}\left(x_{1}-x_{1}^{(j)}\right)=2 m \neq 0 .
$$

Can we put an upper bound on the number of zeros of $\nabla F(\mathbf{x})$ ? In the general case the answer is no.

THEOREM 3. For each integer $n \geqslant 1$ there is a set of $m=n+1$ points $P_{0}, P_{1}, \ldots, P_{n}$, and a corresponding set of functions $g_{j}(r)$ satisfying the conditions of Theorem 1, such that $\nabla F(\mathbf{x})$ has infinitely many zeros in $H$.

Proof. For simplicity, we first consider $n=2$ (the plane) and select the three points $P_{0}(0,0), P_{1}(1,0)$ and $P_{2}(0,1)$. We set

$$
g_{1}(r)=g_{2}(r)=-r+\sqrt{2 r-1}
$$

for $r$ in the interval $J: \varepsilon+\frac{1}{2} \leqslant r \leqslant 1-\varepsilon$. Outside this interval we may define $g_{1}(r)$ and $g_{2}(r)$ in any manner that satisfies the conditions of Theorem 1. We note that in $J$ we have $g_{1}^{\prime}(r)=g_{2}^{\prime}(r)=-1+1 /(2 r-1)^{1 / 2}>0$.

Finally, we let $g_{0}(r)=2 r$. With these selections,

$$
F(\mathbf{x})=\sum_{j=0}^{2} g_{j}\left(r_{j}^{2}\right)=2 r_{0}^{2}-r_{1}^{2}+\sqrt{2 r_{1}^{2}-1}-r_{2}^{2}+2 \sqrt{2 r_{2}^{2}-1},
$$

where $r_{0}^{2}=2\left(x_{1}^{2}+x_{2}^{2}\right), r_{1}^{2}=\left(x_{1}-1\right)^{2}+x_{2}^{2}$ and $r_{2}^{2}=x_{1}^{2}+\left(x_{2}-1\right)^{2}$. Then, for $r_{1}^{2}$ and $r_{2}^{2}$ in $J$,

$$
\begin{gathered}
F(\mathbf{x})=g_{0}\left(r_{0}^{2}\right)+g_{1}\left(r_{1}^{2}\right)+g_{2}\left(r_{2}^{2}\right) . \\
\frac{1}{2} \nabla F(\mathbf{x})=2 x_{1} \mathbf{e}_{1}+2 x_{2} \mathbf{e}_{2}+g_{1}^{\prime}\left(r_{1}^{2}\right)\left[\left(x_{1}-1\right) \mathbf{e}_{1}+x_{2} \mathbf{e}_{2}\right] \\
+g_{2}^{\prime}\left(r_{2}^{2}\right)\left[x_{1} \mathbf{e}_{1}+\left(x_{2}-1\right) \mathbf{e}_{2}\right] .
\end{gathered}
$$

Now consider the line segment $L: x_{1}=x_{2}=t$, where $\delta_{1} \leqslant t \leqslant \frac{1}{2}-\delta_{2}$. Here $\delta_{1}$ and $\delta_{2}$ are small positive constants that depend on $\varepsilon$ and are determined by the requirement that $L$ must lie in that part of the triangle $P_{0} P_{1} P_{2}$ for which $r_{1}^{2}$ and $r_{2}^{2}$ lie in $J$. On the line segment $L$ we find that

$$
g_{1}^{\prime}\left(r_{1}^{2}\right)=g_{2}^{\prime}\left(r_{2}^{2}\right)=2 t /(1-t)
$$

and hence,

$$
\begin{aligned}
\frac{1}{2} \nabla F(\mathbf{x})= & 2 t \mathbf{e}_{1}+2 t \mathbf{e}_{2}+\frac{2 t}{1-2 t}\left[(t-1) \mathbf{e}_{1}+t \mathbf{e}_{2}\right] \\
& +\frac{2 t}{1-2 t}\left[t \mathbf{e}_{1}+(t-1) \mathbf{e}_{2}\right]=0 \mathbf{e}_{1}+0 \mathbf{e}_{2} .
\end{aligned}
$$

Thus, $\nabla F(\mathbf{x})$ is zero for every point of $L$ and, hence, has an uncountable infinity of zeros in $H$.

We observe that each of these infinitely many zeros of $\nabla F(\mathbf{x})$ is a simple zero. Indeed, if $G_{1}(\mathbf{x})$ is the coefficient of $\mathbf{e}_{1}$ in (14), then

$$
\begin{aligned}
\frac{\partial G_{1}(\mathbf{x})}{\partial x_{1}}= & 2+g_{1}^{\prime}\left(r_{1}^{2}\right)+g_{1}^{\prime \prime}\left(r_{1}^{2}\right) 2\left(x_{1}-1\right)^{2} \\
& +g_{2}^{\prime}\left(r_{2}^{2}\right)+g_{2}^{\prime \prime}\left(r_{2}^{2}\right) 2 x_{1}^{2} .
\end{aligned}
$$


A brief computation shows that, on $L$,

$$
\begin{aligned}
\frac{\partial G_{1}(\mathbf{x})}{\partial x_{1}} & =2+\frac{2 t}{1+2 t}-\frac{2(t-1)^{2}}{(1-2 t)^{3}}+\frac{2 t}{(1-2 t)^{3}}-\frac{2 t^{2}}{(1-2 t)^{3}} \\
& =\frac{4 t(t-1)}{(1-2 t)^{3}} \neq 0 .
\end{aligned}
$$

To complete the proof of Theorem 3 we merely indicate the extension to $E_{n}$ for $n>2$.

Let $P_{j}$ be the point with 1 for the $j$ th coordinate and 0 for the other coordinates $(j=1,2, \ldots, n)$. Let $P_{0}$ be the point $(0,0, \ldots, 0)$. We set

$$
g_{j}(r)=-n r+2 \sqrt{n r-(n-1)}, \quad j=1,2, \ldots, n,
$$

for $r$ in the interval $J: \varepsilon+1-1 / n \leqslant r \leqslant 1-\varepsilon$, where $\varepsilon<1 / 2 n$ is a positive number. Outside $J$ we may define $g_{j}(r)$ in any manner that satisfies the conditions of Theorem 1. In $J, g_{j}^{\prime}(r)=-1+(n r-n+1)^{-1 / 2}>0$. Finally, we set $g_{0}(r)=n^{2} r$. Then

$$
\begin{gathered}
F(\mathbf{x})=\sum_{j=0}^{n} g_{j}\left(r_{j}^{2}\right)=n^{2} r_{0}^{2}+\sum_{j=1}^{n}\left[-n r_{j}^{2}+2 \sqrt{n r_{j}^{2}-n+1}\right] . \\
\nabla F(\mathbf{x})=n^{2} \nabla r_{0}^{2}+\sum_{j=1}^{n} g_{j}^{\prime}\left(r_{j}^{2}\right) \nabla r_{j}^{2} \equiv \sum_{k=1}^{n} G_{k}(\mathbf{x}) \mathbf{e}_{k} .
\end{gathered}
$$

Now consider the line segment $L: x_{1}=x_{2}=\cdots=x_{n}=t$, where $\delta_{1} \leqslant t$ $\leqslant 1 / n-\delta_{2}$ and $\delta_{1}$ and $\delta_{2}$ are small positive numbers that depend on $\varepsilon$. On this line segment,

$$
\begin{gathered}
\nabla r_{0}^{2}=2 t \sum_{k=1}^{n} \mathbf{e}_{k}, \\
\nabla g_{j}\left(r_{j}^{2}\right)=\frac{2 n^{2} t}{1-n t}\left[-\mathbf{e}_{j}+\sum_{k=1}^{n} t \mathbf{e}_{k}\right], \quad j=1,2, \ldots, n .
\end{gathered}
$$

Consequently, for each component in (20), we find that

$$
G_{k}(\mathbf{x})=2 t n^{2}+n \frac{2 n^{2} t^{2}}{1-n t}-\frac{2 n^{2} t}{1-n t} \equiv 0 .
$$

3. Further questions. (1) The application of the Brouwer fixed point theorem in the proof of Theorem 1 requires that $g_{j}^{\prime}(r)$ be continuous in $r \geqslant 0$. Further, we asked that $g_{j}^{\prime}(r)$ be positive for $r \geqslant 0$. Of course this latter condition can be weakened to $g_{j}^{\prime}(r) \geqslant 0$, as long as we have $\sum_{j=1}^{m} g_{j}^{\prime}\left(r_{j}^{2}\right)>0$.

If $g_{j}^{\prime}(r) \rightarrow \infty$ as $r \rightarrow 0$, the proof of Theorem 1 is still valid because the factors $B_{j}$ in equation (9) will still be continuous in $H$. However, if we permit $g_{j}^{\prime}(r)$ to have a discontinuity of some other type, then the existence of a zero of $\nabla F(\mathbf{x})$ is an open question.

(2) In Theorem 3 we saw that $\nabla F(\mathbf{x})$ can be zero on a line segment-a set of positive linear measure. Is it possible to have $\nabla F(\mathbf{x})=\mathbf{0}$ on some set that has $q$-dimensional positive measure, where $q>1$ ? 
(3) If the answer in (2) is no, is it possible to have $\nabla F(\mathbf{x})=\mathbf{0}$ on an arbitrary curve?

The author feels that the answer in (2) is no, and that the conditions on $F(\mathbf{x})$ put a severe limit on the shape of the curve on which $\nabla F(\mathbf{x})$ may vanish.

(4) To obtain $\nabla F(\mathbf{x})=\mathbf{0}$ on a line segment, we selected two of the functions $g_{j}(r)$ to be different. Suppose that in equation (5) each $g_{j}$ is the same function. Is it then true that $F(x)$ has only a finite number of zeros? If so, is this number $\leqslant m-1$ ?

\section{REFERENCES}

1. J. B. Diaz and Dorothy Browne Shaffer, $A$ generalization to higher dimensions of a theorem of Lucas concerning the zeros of the derivative of a polynomial of one complex variable, Proc. Internat. Congress Math. (Vancouver, 1974), Canad. Math. Congress (to appear).

2. N. Dunford and J. T. Schwartz, Linear operators. I: General theory, Pure and Appl. Math., vol. 7, Interscience, New York, 1958. MR22 \#8302.

3. Morris Marden, Geometry of polynomials, Math. Surveys, no. 3, Amer. Math. Soc., Providence, R.I., 1966. MR27 \#1562.

4. G.V. Sz.-Nagy, Über die Lage der Nullstellen eines Abstandspolynoms and seiner Derivierten, Bull. Amer. Math. Soc. 55(1949), 329-342. MR10, 702.

5. A. Schurrer, On the location of the zeros of the derivative of rational functions of distance polynomials, Trans. Amer. Math. Soc. 89(1958), 100-112. MR20 \#4634.

Department of Mathematics, University of South Florida, Tampa, Forida 33620 